\title{
Super-Resolution Ultrasound Imaging of Rat Kidneys before and after Ischemia- Reperfusion
}

Bech Andersen, Sofie ; Villagómez Hoyos, Carlos Armando; Taghavi, Iman; Granberg, Fredrik; Hansen, Kristoffer Lindskov; Sørensen, Charlotte Mehlin; Jensen, Jørgen Arendt; Nielsen, Michael Bachmann

\section{Published in:}

Proceedings of 2019 IEEE International Ultrasonics Symposium

Link to article, DOI:

10.1109/ULTSYM.2019.8926190

Publication date:

2019

Document Version

Peer reviewed version

Link back to DTU Orbit

Citation (APA):

Bech Andersen, S., Villagómez Hoyos, C. A., Taghavi, I., Granberg, F., Hansen, K. L., Sørensen, C. M., Jensen, J. A., \& Nielsen, M. B. (2019). Super-Resolution Ultrasound Imaging of Rat Kidneys before and after IschemiaReperfusion. In Proceedings of 2019 IEEE International Ultrasonics Symposium (pp. 1169-1172). IEEE. https://doi.org/10.1109/ULTSYM.2019.8926190

\section{General rights}

Copyright and moral rights for the publications made accessible in the public portal are retained by the authors and/or other copyright owners and it is a condition of accessing publications that users recognise and abide by the legal requirements associated with these rights.

- Users may download and print one copy of any publication from the public portal for the purpose of private study or research.

- You may not further distribute the material or use it for any profit-making activity or commercial gain

- You may freely distribute the URL identifying the publication in the public portal 


\title{
Super-Resolution Ultrasound Imaging of Rat Kidneys before and after Ischemia-Reperfusion
}

\author{
Sofie Bech Andersen ${ }^{1,2,3}$, Carlos Armando Villagómez Hoyos ${ }^{4}$, Iman Taghavi ${ }^{5}$, Fredrik Gran ${ }^{4}$, \\ Kristoffer Lindskov Hansen ${ }^{1,3}$, Charlotte Mehlin Sørensen ${ }^{2}$, Jørgen Arendt Jensen ${ }^{5}$, Michael Bachmann Nielsen ${ }^{1,3}$ \\ ${ }^{1}$ Department of Diagnostic Radiology, Rigshospitalet, Copenhagen, Denmark. \\ ${ }^{2}$ Department of Biomedical Sciences, University of Copenhagen, Copenhagen, Denmark. \\ ${ }^{3}$ Department of Clinical Medicine, University of Copenhagen, Copenhagen, Denmark. \\ ${ }^{4}$ BK Medical ApS, Herlev, Denmark. \\ ${ }^{5}$ Center for Fast Ultrasound, Department of Health Technology, Technical University of Denmark, Lyngby, Denmark.
}

\begin{abstract}
In vivo visualization of the microvasculature is feasible with super-resolution ultrasound imaging (SRI), but the method needs more affirmative data before clinical use. The kidneys have a rich vasculature, and microvascular dysfunction decreases the kidney function. Therefore, detection of subtle renal microvascular changes could benefit patients with renal disease. We hypothesized that our SRI setup can visualize the microvascular network of two healthy rat kidneys and subsequently demonstrate microvascular flow changes immediately after ischemiareperfusion. The left kidney of two male Sprague-Dawley rats was scanned during laparotomy using a customized BK5000 scanner, an X18L5s transducer, and interleaved contrast and B-mode sequences with focused beam transmission. Images were acquired over 10 min using SonoVue (1:10) as the contrast agent. After a baseline scan, one rat had the renal vein clamped, while the other rat had the renal artery clamped, both for $\mathbf{4 5} \mathrm{min}$. The kidneys were rescanned immediately after clamp release and after $60 \mathrm{~min}$ of reperfusion. Motion correction was applied before microbubble (MB) detection. The characteristic renal microvascular structure was visualized with anatomical distinction between the dense cortical vascular network and the straight vessels of the medulla. Immediately after vein clamp release, almost no MBs perfused the medullary vessels. After $60 \mathrm{~min}$ of reperfusion, MBs refilled the renal vascular bed, but with a slower velocity compared with the baseline scan. The results after artery clamping were subtler, with more MBs perfusing the medulla immediately after clamp release compared with the vein clamping. The results imply that this SRI setup can evaluate different stages of ischemic kidney disease in rats as it can visualize the entire renal vascular bed and differentiate the pattern of reperfusion in two types of ischemic injury.
\end{abstract}

\section{INTRODUCTION}

The microvasculature of organs and tissues, including the kidneys of rats and rabbits, has been visualized in vivo with super-resolution ultrasound imaging (SRI) [1]-[4]. However, there are few studies concerning pathological changes in the microvasculature detected by SRI. SRI is based on the nonlinear behavior of gas-filled microbubbles (MBs) injected into the bloodstream. By the use of a series of pulse transmissions, the echoes from individual MBs can be separated from the surrounding linearly-behaving tissue. The position of each MB centroid is accumulated over many frames. By processing a stack of frames, an image of the microvascular network is formed, and estimations of the MB velocity and direction can be made [1], [5]. The kidneys are highly vascular organs, and microvascular dysfunction decreases the kidney function considerably. Detection of modest changes in the structure or function of the renal microvasculature could improve diagnostics, treatment possibilities, and prognosis for patients with diseases such as ischemic kidney disease or diabetic nephropathy. Renal ischemia-reperfusion injuries are caused by a temporary loss of blood flow and can lead to acute kidney injury with a rapid loss of kidney function [6]. Ischemiareperfusion injuries are often used as models for acute kidney injury in rodents. The injuries are induced by placing a microclamp on the renal artery, vein, or pedicle for 30-60 min with subsequent release and reperfusion. The renal vein clamp causes the severest tissue damage [7]. Is has been shown that the blood flow in the kidneys is reduced after ischemia with subsequent reperfusion [8]-[10].

Using SRI, we aimed to visualize the renal microvasculature of two healthy male Sprague-Dawley rats and show subsequent pathological microvascular flow changes immediately after unilateral renal ischemia-reperfusion.

\section{MATERIALS AND METHODS}

\section{A. Animal preparation}

We conducted the experiment on two healthy male SpragueDawley rats (body weight 251 and $357 \mathrm{~g}$ ). The experiments were performed according to protocols approved by the Danish National Animal Experiments Inspectorate. The procedures were performed at the University of Copenhagen, and all local ethical standards were followed. The ethical policy of the university complies with that of the National Institutes of Health $(\mathrm{NIH})$. All animals were housed in the animal facility at the University of Copenhagen (Department of Experimental Medicine), where trained animal caretakers were responsible for the animal welfare. Anesthesia was induced with $5 \%$ isoflurane. After tracheotomy, the animals were connected to a mechanical 
ventilator with a respiration cycle of 72 respirations $/ \mathrm{min}$. A continuous infusion of Nimbex (cisatracurium, $0.85 \mathrm{mg} / \mathrm{ml}$, GlaxoSmithKline) was given, and adequate anesthesia was maintained with 1-2\% isoflurane. Polyethylene catheters (PE10) were used for jugular vein catheterization and subsequent infusion of MBs (SonoVue, Bracco), isotonic saline, and Nimbex. The arterial pressure was monitored with a polyethylene catheter (PE-50) in the left carotid artery. The animals were placed in the supine position on a heating pad, and the left kidney was exposed through laparotomy. The left side of the diaphragm was pulled slightly in the cranial direction with a metal retractor to further expose the kidney and reduce respiratory motion. The kidney was kept warm with heated isotonic saline in-between ultrasound scans. After the last scan, the rats were euthanized.

\section{B. Ultrasound scan procedure and SRI technique}

A costumized BK5000 scanner and a fixed X18L5s transducer (BK Medical ApS, Herlev, Denmark) were used to obtain data. The transducer was placed on the lateral surface of the left kidney, using ultrasound gel for coupling. The MBs were diluted in isotonic saline $(1: 10)$ and infused intravenously at $100 \mu \mathrm{l} / \mathrm{min}$. The $10-\mathrm{min}$ data recording started when the MBs appeared on the scanner display. To avoid floating of MBs in the syringe, a custom-built device turned the syringe $180^{\circ}$ every 60 sec. After a baseline SRI scan, Rat 1 had the renal vein clamped, and Rat 2 had the renal artery clamped using a nontraumatic micro-clamp. After $45 \mathrm{~min}$, the clamp was removed and immediately afterward, a second SRI scan was done. The last SRI scan was performed 60 min after clamp release. Before each scan, the imaging plane was readjusted with reference to the baseline B-mode scan. As a consequence, the three imaging planes were not identical. Power Doppler ultrasound (PRF: $400 \mathrm{~Hz}$, wall filter cutoff: $80 \mathrm{~Hz}$ ) was acquired immediately after the first and third SRI scans for comparison. The MBs were insonified by interleaved B-mode and contrast-enhancing sequences (amplitude modulation) with line-per-line focused beam transmissions (frame rate: $54 \mathrm{~Hz}$, center frequency: $6 \mathrm{MHz}$, mechanical index: 0.2). Envelope Bmode data were used to estimate lateral and axial motion relative to a reference frame by tissue speckle tracking [11]. Motion was compensated using the motion estimation to adjust the position of each MB back to its location on the reference

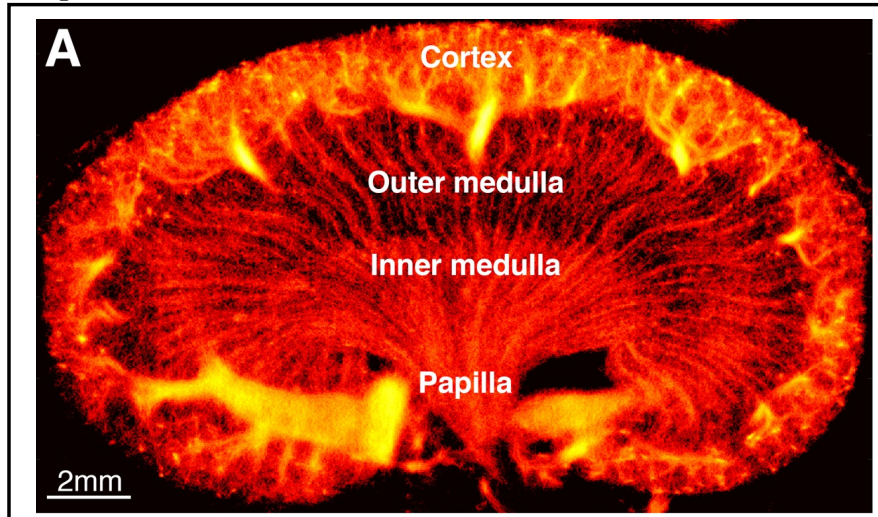

frame. MB tracks were made by connecting the nearest MBs in consecutive images. The tracks were subsequently inserted in the high-resolution images to yield MB track density images, and the estimated $\mathrm{MB}$ velocities were used to create the superresolution velocity images. To estimate both cortical and medullary blood flow, while also accounting for regional differences in ischemic damage, the MB velocity estimations were calculated for MBs flowing toward the renal surface (primarily interlobular arteries) in three separate cortical regions of interest (ROIs) and MBs flowing toward the papilla (descending straight vessels) in three separate ROIs of the outer medulla. The MB velocity was estimated for all the tracks in the ROIs. Additionally, a perfusion index, Q, was calculated:

$$
\text { Perfusion index, } Q=\bar{v} \cdot A \text {, }
$$

where $\bar{v}$ is the mean velocity for all the tracks in the ROI and $A$ is the area of the ROI.

\section{Histopathology}

After euthanasia, the left kidney was removed and fixated in $4 \%$ paraformaldehyde for documentation of the tissue damage. A sham kidney that had undergone three SRI scans but no ischemic injury was also removed and fixated. The kidneys were embedded in paraffin, cut into $4 \mu \mathrm{m}$ slices, and stained using a standard hematoxylin and eosin (H\&E) staining protocol. The images were analyzed by a trained and blinded anatomist.

\section{RESULTS}

\section{A. The microvasculature of the healthy rat kidneys}

In the healthy kidneys, a mean of $106 \mathrm{MBs} /$ frame (Rat 1) and $59 \mathrm{MBs} /$ frame (Rat 2) were detected (32,300 frames/scan). The track density images revealed the anatomical structure of the renal vasculature with a clear distinction between the dense cortical vascular network and the straight vessels (vasa recta) of the outer and inner medulla (Fig. 1-A). As shown by the color wheel in Fig. 1-B, the velocity maps revealed opposing flow directions in adjacent arteries and veins, allowing further discrimination between vessels that were not separable on the track density images. The mean MB velocities in the descending straight vessels of the outer medulla in the two rats were $1.33 \pm 0.05$ and $1.15 \pm 0.09 \mathrm{~mm} / \mathrm{sec}$, respectively. These velocities

Fig. 1. A) Track density image (log scale, 3 decades) of Rat 1 showing the normal renal microvasculature. B) Microbubble velocity image showing the microbubble flow directions according to the color wheel (top right). This enables distinction between arteries and veins; here examplified by the distinction between the descending (DSV) and ascending (ASV) straight vessels. The image is filtered and includes only tracks longer than $250 \mu \mathrm{m}$ with a max velocity of $3.0 \mathrm{~mm} / \mathrm{sec}$ to highlight the medullary straight vessels.

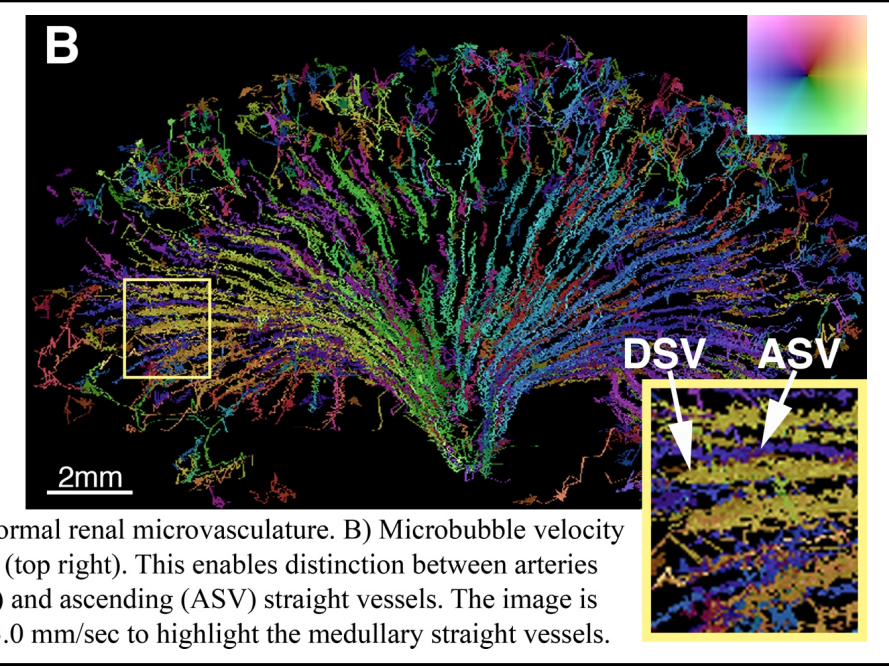


were calculated only for vessels longer than $500 \mu \mathrm{m}$ to increase the accuracy of the estimations.

\section{B. Microvascular changes after renal ischemia-reperfusion}

Changes in the renal microvasculature after ischemiareperfusion were visible and measurable with SRI. During the first $10 \mathrm{~min}$ of reperfusion after clamping of the renal vein, the entire kidney was poorly perfused with MBs compared with the baseline scan (mean of $55 \mathrm{MBs} /$ frame vs. $106 \mathrm{MBs} /$ frame at baseline) (Fig. 2-A1). This was especially evident in the medulla where almost no MBs were detected. The mean of the estimated MB velocity and perfusion index in the ROIs was also lower compared with baseline (perfusion index shown in Fig. 2-A2). The results were similar, but subtler immediately after the artery clamping with some MB perfusion of the medullary vessels (Fig. 2-B1, mean of $29 \mathrm{MBs} /$ frame vs. $59 \mathrm{MBs} /$ frame at baseline). After $60 \mathrm{~min}$ of reperfusion, MBs refilled the entire renal vascular bed again (Fig. 2-A1 and -B1), with a mean of 89 $\mathrm{MBs} /$ frame after vein clamping and $50 \mathrm{MBs} /$ frame after artery clamping. The vasculature appeared more irregular on the track density images after clamping. After vein clamping, the mean of the estimated $\mathrm{MB}$ velocity and perfusion index increased again, but to a lower level than baseline. After artery clamping, the mean of the estimated $\mathrm{MB}$ velocity and perfusion index remained low. However, there were differences among the individual ROIs in the response to the injuries, as seen in Fig. 2-A2 and -B2. The power Doppler ultrasound did not visualize the medullary blood flow. After $60 \mathrm{~min}$ of reperfusion, power Doppler showed a decreased cortical blood flow compared with baseline in both kidneys. The H\&E stains showed signs of acute tubular necrosis in both specimens. Additionally, a large number of red blood cells were accumulated interstitially after vein clamping (Fig. 3). These changes were not present in the sham kidney.

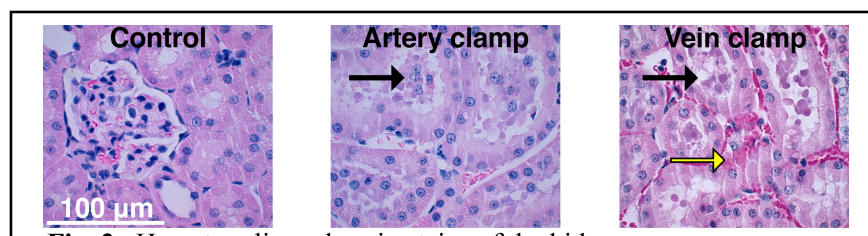

Fig. 3. Hematoxylin and eosin stains of the kidneys.

Black arrows: Intratubular cast formations.

Yellow arrow: Red blood cells accumulated in the interstitium.

\section{DISCUSSION AND CONCLUSION}

In this small preclinical study, we investigated the ability of the presented SRI setup to visualize the microvasculature of two healthy rat kidneys. We found a detailed anatomical depiction of the renal vascular tree similar to descriptions from early anatomical studies [12] and nano-CT images of corrosion casts of mouse kidneys [13]. The mean $\mathrm{MB}$ velocities in the descending straight vessels are comparable to the $0.94 \pm 0.24$ $\mathrm{mm} / \mathrm{sec}$ found by Foiret et al. [2]. The early effects of renal ischemia-reperfusion were also investigated: Immediately after vein clamp release, the SRI showed a more pronounced reduction in the refilling of the medullary vessels compared with the artery clamping. Visually and with B-mode ultrasound it was observed that the renal vein clamp caused subcapsular edema. Also, the H\&E stains revealed a large amount of red blood cells clotted in the renal interstitial space. These findings are in accordance with a previous study that reported vein occlusion to inflict more tissue damage than artery occlusion [7]. The significant reduction in the refilling of the vessels is most likely due to compression, caused by the interstitial edema and accumulated red blood cells. Even though MBs returned to the renal vascular bed after $60 \mathrm{~min}$ of reperfusion, the MB velocity and perfusion index were lower compared with baseline; this is also in accordance with previous studies [8]-[10] and with our

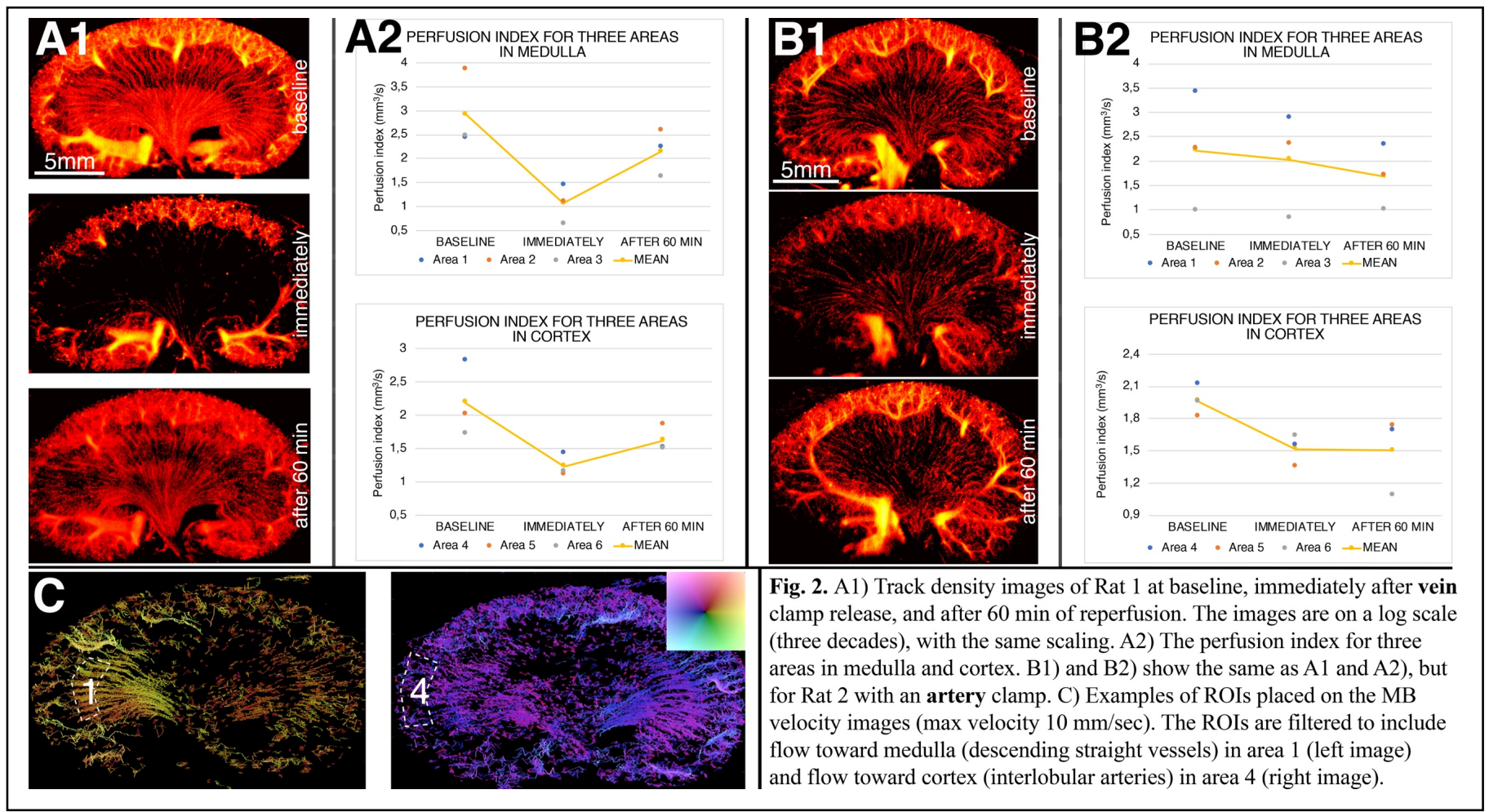


power Doppler scans. Despite the differences in reperfusion immediately after clamp release and the differences between the H\&E stains, a direct distinction between the 60 min SRI scans of the two rats could not be made. Also, the MB count differed between the two kidneys at baseline, making a direct comparison difficult. Nevertheless, this is - to our knowledge - the first time SRI has shown pathological changes in MB velocity in the small vessels of the outer renal medulla as well as the first time it has differentiated the reperfusion pattern after two types of ischemia-reperfusion.

Our estimations of the mean $\mathrm{MB}$ velocity and perfusion index in the cortex and medulla are not comparable: The renal cortex receives approximately $90 \%$ of the total renal blood flow and has a dense and complex microvascular network. This makes microbubble tracking and $\mathrm{MB}$ velocity estimation difficult, which is exemplified in Fig. 2-C: There are fewer cortical tracks that represent interlobular arteries than expected. Generally, the interlobular veins seem easier to track. It could be because the veins are larger than the arteries and sometimes partly encircle the arteries, thereby hindering the tracking of the MBs in the arteries. The 2D image sections were not identical in the three consecutive scans. This is particularly apparent for Rat 2 (Fig. 2-B1). The difference in the image sections poses a potential issue with the comparison of the consecutive scans: The differences in MB velocity and perfusion index between the ROIs may represent physiologically determined differences, but may also result from a direct comparison of anatomically unidentical regions. That issue can be eliminated with 3D SRI. 3D SRI can also solve other limitations of 2D SRI: It will allow tracking of MBs that move in the elevation plane, eliminate the risk of missing essential information outside the image section, and allow scans from the skin surface. We scanned directly on the kidney surface to reduce respirational motion and the motion that occurs when neighboring bowels push the kidney out of plane; both of which are problematic for 2D motion correction. The data from this study will be used to further optimize the motion correction algorithm, hopefully making transcutaneous 2D SRI scanning possible. Transcutaneous scanning would make SRI applicable for long-term studies, such as investigation of how or when the microvascular changes in diabetic nephropathy or the late stages of ischemia-reperfusion injuries occur [14], and how these correlate with the early changes in microvascular blood flow. If early changes could be used for prognostic evaluation of patients and allow timely treatment initiation, SRI could become a strong clinical tool.

Plane-wave techniques are often used in SRI to obtain images at a very high framerate [1]-[4], [9]. We used focused beam transmission, because it is a well-established technology already available in commercial platforms. This could enable a faster transfer to the clinic. A disadvantage of focused beam transmission is the lower frame rate and longer acquisition time. Generally, time consumption is an often-emphasized limitation of SRI, but in order to generate super-resolution images of a complete microvascular network using MBs, the acquisition time has to be lengthy. The MBs follow the blood flow, which is slow in the capillaries, and a sufficient amount of MB detections is needed to create the images [15]. Also, compared with other imaging modalities such as MRI that is expensive and less accessible or CT that poses a radiation hazard, the time spent attaining ultrasound images of such high resolution should be acceptable when applied to the appropriate clinical settings.

In conclusion, this small study indicates that our SRI setup can be used to evaluate not only different stages of ischemic kidney disease, but also other renal diseases, since the method depicts all areas of the renal microvasculature and can detect differences in reperfusion after two different ischemiareperfusion events. However, the experiments were performed using only two rats. A more extensive study will be conducted.

\section{ACKNOWLEDGMENTS}

Thanks to Henrik Koch Johansen (BK Medical ApS) for constructing the syringe-turning device. Financial study support: Innovation Fund Denmark (grant 7050-00004B).

\section{REFERENCES}

[1] C. Errico et al., 'Ultrafast ultrasound localization microscopy for deep super-resolution vascular imaging.', Nature, vol. 527, no. 7579, pp. 499502, Nov. 2015.

[2] J. Foiret, H. Zhang, T. Ilovitsh, L. Mahakian, S. Tam, and K. W. Ferrara, 'Ultrasound localization microscopy to image and assess microvasculature in a rat kidney.', Sci. Rep., vol. 7, no. 1, p. 13662, Oct. 2017.

[3] J. Yu, L. Lavery, and K. Kim, 'Super-resolution ultrasound imaging method for microvasculature in vivo with a high temporal accuracy', Sci. Rep., vol. 8, no. 1, p. 13918, Dec. 2018.

[4] T. Opacic et al., 'Motion model ultrasound localization microscopy for preclinical and clinical multiparametric tumor characterization.', Nat. Commun., vol. 9, no. 1, p. 1527, 2018.

[5] K. Christensen-Jeffries, R. J. Browning, M. X. Tang, C. Dunsby, and R. J. Eckersley, 'In vivo acoustic super-resolution and super-resolved velocity mapping using microbubbles', IEEE Trans. Med. Imaging, vol. 34, no. 2, pp. 433-440, Feb. 2015.

[6] A. S. Levey and M. T. James, 'Acute Kidney Injury', Ann. Intern. Med., vol. 167, no. 9, p. ITC66, Nov. 2017.

[7] S. M. Owji, E. Nikeghbal, and S. M. Moosavi, 'Comparison of ischaemia-reperfusion-induced acute kidney injury by clamping renal arteries, veins or pedicles in anaesthetized rats', Exp. Physiol., vol. 103, no. 10 , pp. $1390-1402$, Oct. 2018.

[8] W. Cao et al., 'Contrast-Enhanced Ultrasound for Assessing Renal Perfusion Impairment and Predicting Acute Kidney Injury to Chronic Kidney Disease Progression', Antioxid. Redox Signal., vol. 27, no. 17, pp. 1397-1411, Dec. 2017.

[9] Y. Yang et al., 'Assessment of Diabetic Kidney Disease Using Ultrasound Localization Microscopy: An in Vivo Feasibility Study in Rats', in Proc. IEEE Ultrason. Symp., 2018, pp. 1-4.

[10] K. Fischer et al., 'High-resolution renal perfusion mapping using contrast-enhanced ultrasonography in ischemia-reperfusion injury monitors changes in renal microperfusion.', Kidney Int., vol. 89, no. 6, pp. 1388-98, 2016.

[11] J. A. Jensen, S. B. Andersen, C. A. V. Hoyos, K. L. Hansen, C. M. Sørensen, and M. B. Nielsen, 'Tissue Motion Estimation and Correction in Super Resolution Imaging', in Proc. IEEE Ultrason. Symp., 2019.

[12] D. B. Moffat and J. Fourman, 'A vascular pattern of the rat kidney. 1963.', J. Am. Soc. Nephrol., vol. 12, no. 3, pp. 624-32, Mar. 2001.

[13] R. Wagner, D. Van Loo, F. Hossler, K. Czymmek, E. Pauwels, and L. Van Hoorebeke, 'High-Resolution Imaging of Kidney Vascular Corrosion Casts with Nano-CT', Microsc. Microanal., vol. 17, no. 2, pp. 215-219, Apr. 2011.

[14] H.-J. Wang, A. Varner, T. AbouShwareb, A. Atala, and J. J. Yoo, 'Ischemia/Reperfusion-Induced Renal Failure in Rats as a Model for Evaluating Cell Therapies', Ren. Fail., vol. 34, no. 10, pp. 1324-1332, Nov. 2012.

[15] V. Hingot, C. Errico, B. Heiles, L. Rahal, M. Tanter, and O. Couture, 'Microvascular flow dictates the compromise between spatial resolution and acquisition time in Ultrasound Localization Microscopy.', Sci. Rep., vol. 9, no. 1, p. 2456, Feb. 2019. 